\title{
Analysis of the Nonlinear Maxwell-Cattaneo-Vernotte Equation ${ }^{+}$
}

\author{
Róbert Kovács ${ }^{1}$ and Patrizia Rogolino ${ }^{2}$ \\ 1 Department of Energy Engineering, BME, Budapest, Hungary \\ 2 Università degli Studi di Messina, Messina, Italy \\ + Presented at the Entropy 2021: The Scientific Tool of the 21st Century, 5-7 May 2021; Available online: \\ https://sciforum.net/conference/Entropy2021/.
}

Published: 5 May 2021

It is well-known that the Fourier equation for heat conduction is not satisfactory in many cases, such as low-temperature situations. It motivated the researchers to find possible extensions. There are numerous approaches in the literature, here we apply non-equilibrium thermodynamics with internal variables.

The first, and successfully applied generalized constitutive equation is called Maxwell-CattaneoVernotte $(\mathrm{MCV})$ equation. It is quite straightforward to derive using the internal variable theory. However, when nonlinear attributes come into the picture, there are some significant consequences that must be investigated further.

In the present paper [1], we are considering temperature-dependent material parameters, e.g., the thermal conductivity and the relaxation time both depend on the temperature. A consistent analysis shows that in some cases, the temperature dependence of mass density follows immediately. It cannot be avoided; thus, the mechanical field has to be introduced to obtain a physically admissible solution.

On the other hand, we investigated the numerical solutions of such a nonlinear MCV equation. We found that the nonlinear numerical stability analysis can be substituted with the linear one by estimating the maximum of the temperature field apriori. Here, we present the effects of temperature dependence and demonstrating the usage of the developed numerical code.

\section{Reference}

1. Kovács, R.; Rogolino, P. Numerical treatment of nonlinear Fourier and Maxwell-Cattaneo-Vernotte heat transport equations. Int. J. Heat Mass Transf. 2020, 150, 119281.

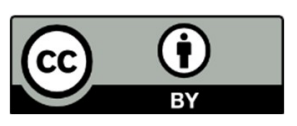

(C) 2021 by the authors. Licensee MDPI, Basel, Switzerland. This article is an open access article distributed under the terms and conditions of the Creative Commons Attribution (CC BY) license (http://creativecommons.org/licenses/by/4.0/). 